Dossier: La escritura en los desplazamientos: viajes, expediciones y naufragios

\title{
La caracterización del clima en el espacio geográfico costarricense visto desde la perspectiva de ocho autores extranjeros (1888-1944)
}

\author{
Ronald Eduardo Díaz Bolaños \\ Universidad Estatal a Distancia, Costa Rica \\ ronald.diaz@ucr.ac.cr \\ http://orcid.org/0000-0003-2860-6744
}

Recibido: 14 de febrero de 2020

Aceptado: 30 de marzo de 2020

Resumen: El conocimiento sobre el clima en el espacio geográfico en el que se asienta la República de Costa Rica fue una labor desarrollada por instituciones científicas a lo largo del siglo XIX y la primera mitad del siglo XX. Durante este proceso colaboraron numerosos científicos y estudiosos que plasmaron en sus obras descripciones sobre las condiciones climáticas del país, fruto de su trabajo científico o de sus experiencias personales. Los autores seleccionados fueron ocho: Henri Pittier, Karl Sapper, Dana G. Munro, Max Sorre y Rosa Filatti, Charles Borel y John y Mavis Biesanz. El período de estudio inicia en 1888 con la publicación de "Apuntaciones sobre el clima é hipsometría de la República de Costa Rica. I. Resumen de las observaciones meteorológicas anteriores al año de 1888" de Pittier y concluye en 1944, año en que se publica la obra Costa Rican Life de John y Mavis Biesanz. Este período coincide con el denominado "régimen de cientificidad", una etapa del desarrollo científico costarricense en que el Estado, la comunidad científica y el sector privado, confluyen sus intereses para fomentar el desarrollo de la ciencia en el país. Esto precisamente cuando se consolida el modelo agroexportador donde el conocimiento del clima era fundamental para un mejor desempeño de las actividades productivas.

Palabras claves: Costa Rica, historia, ciencia, clima, viajeros.

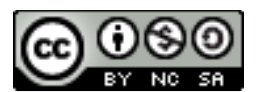

La Revista Estudios es editada por la Universidad de Costa Rica y se distribuye bajo una Licencia Creative Commons Atribución-NoComercial-Compartirlgual 3.0 Costa Rica. Para más información envíe un mensaje a 
Dossier: La escritura en los desplazamientos: viajes, expediciones y naufragios

\title{
The characterization of the climate in the Costa Rican geographical space studied from the perspective of eight foreign authors (1888-1944)
}

\begin{abstract}
The knowledge about the climate in the geographical space in which the Republic of Costa Rica is based was developed by scientific institutions throughout the 19th century and the first half of the 20th century. During this process many scientists and scholars collaborated with essays that reflected descriptions of the climatic conditions of the country, as a result of their scientific inquiries or personal experiences. The authors selected were eight: Henri Pittier, Karl Sapper, Dana G. Munro, Max Sorre and Rosa Filatti, Charles Borel and John and Mavis Biesanz. The period of study begins in 1888 with the publication of "Apuntaciones sobre el clima é hipsometría de la República de Costa Rica. I. Resumen de las observaciones meteorológicas anteriores al año de 1888" by Pittier and concludes in 1944, the year concerning the publication of the work Costa Rican Life by John and Mavis Biesanz. This period concurs with the so-called "scientific regime", a stage of Costa Rican scientific development in which the State, the scientific community and the private sector converge their interests to promote the development of science in the country. This precisely when the agro-export model is consolidated, and climate acquaintanceship was essential for a better performance of the productive activities.
\end{abstract}

Keywords: Costa Rica, history, science, climate, travelers.

\section{(C) $(0 \bigcirc)$}

La Revista Estudios es editada por la Universidad de Costa Rica y se distribuye bajo una Licencia Creative Commons Atribución-NoComercial-CompartirIgual 3.0 Costa Rica. Para más información envíe un mensaje a 
Dossier: La escritura en los desplazamientos: viajes, expediciones y naufragios

\section{Introducción}

Cuando di comienzo á mis estudios, á penas podía contar con el material que requiere una estación de segundo orden y es lo cierto que ni tampoco disponía en aquel entonces del auxilio con que cuento hoy día para practicar observaciones más completas y de más provecho para el país.

Henri Pittier (1888, p.24).

Costa Rica, un pequeño país que había alcanzado su independencia en 1821, inició su vinculación con el mercado mundial gracias al cultivo del café, proceso que se acrecentó a finales del siglo XIX con la comercialización del banano, lo que hizo posible consolidar una economía agroexportadora basada principalmente en el bicultivismo.

Este proceso permitió a lo largo del siglo XIX el arribo de numerosos científicos, exploradores y viajeros, quienes plasmaron en sus escritos distintos aspectos referentes a la climatología y al paisaje costarricense y fueron fundamentales para la comprensión del estudio del clima en este país centroamericano, además de muchos otros aspectos relacionados con las condiciones políticas, económicas, sociales, culturales y ambientales en los albores de la Costa Rica republicana.

Entre dichos científicos figuraron una pléyade de naturalistas europeos, alemanes en su mayoría, que recorrieron el país y llegaron a dejar valiosos datos de temperatura y precipitaciones para la posteridad y entre los que se puede mencionar a Anders Sandoe Oersted (1816-1872), Moritz Wagner (1813-1887), Karl von Scherzer (1821-1903), Karl Hoffmann (1823-1859), Alexander von Frantzius (18211877), Karl von Seebach (1839-1880) y Helmuth Polakowsky (1847-1917), quienes

\section{(c) (i) (-)}

La Revista Estudios es editada por la Universidad de Costa Rica y se distribuye bajo una Licencia Creative Commons Atribución-NoComercial-CompartirIgual 3.0 Costa Rica. Para más información envíe un mensaje a 
Dossier: La escritura en los desplazamientos: viajes, expediciones y naufragios investigaron en el territorio costarricense entre las décadas de 1840 y 1870 (Solano, 1999, pp.102-138; Hilje, 2013).

La importancia que dieron los gobernantes a los estudios meteorológicos para fomentar las actividades agropecuarias y la apertura de nuevos frentes de colonización, la aparición de una incipiente pero activa comunidad científica y los aportes de los científicos y viajeros extranjeros con sus escritos sobre el clima del país incentivaron los avances de la Meteorología en Costa Rica. Dichos textos tenían la finalidad de dar a conocer al país en las latitudes medias, con el propósito de difundir información sobre sus recursos naturales, fomentar los proyectos de colonización extranjera, gracias a la promoción positiva del clima y su incidencia en la configuración del paisaje costarricense.

Precisamente, el aporte de las investigaciones realizadas por el personal científico extranjero fue uno de los factores que se concatenaron para que el 27 de diciembre de 1887, el Estado autorizara la fundación de un Observatorio Meteorológico en el naciente Liceo de Costa Rica. Esta entidad, sustituyó a la Oficina de Estadística en el acopio de información meteorológica a nivel nacional y sus registros fueron los únicos que recibieron la autorización del Estado costarricense para su publicación y difusión (Solano, Díaz y Amador, 2013, pp.84-94).

Al año siguiente, el Observatorio Meteorológico se amplió al dotársele de estaciones meteorológicas en diferentes puntos del país y pasó a ser la base del Instituto Meteorológico Nacional, entidad que en las siguientes décadas fue integrado a una serie de instituciones científicas y culturales para que continuara con sus actividades en el campo del conocimiento de la atmósfera. Además, sus registros fueron utilizados por científicos e instituciones extranjeras para la elaboración de investigaciones sobre las condiciones climáticas características del territorio

\section{(C) $(\Theta \odot \odot$}

La Revista Estudios es editada por la Universidad de Costa Rica y se distribuye bajo una Licencia Creative Commons Atribución-NoComercial-CompartirIgual 3.0 Costa Rica. Para más información envíe un mensaje a 
Dossier: La escritura en los desplazamientos: viajes, expediciones y naufragios costarricense (Secretaría de Instrucción Pública de la República de Costa Rica, 1889, pp.XIV-XIX; Díaz, 2003, pp.172-311).

El presente trabajo analiza la percepción del clima costarricense descrito por diversos autores extranjeros, quienes plasmaron sus observaciones propias o a partir de otros textos consultados, para comprender la historia del clima y la Meteorología en Costa Rica, en el período que va entre la publicación de "Apuntaciones sobre el clima é hipsometría de la República de Costa Rica" (Pittier, 1888) y Costa Rican Life (Biesanz y Biesanz, 1944). Esta periodización coincide con el ascenso y crisis del Estado liberal y la llegada al poder de gobiernos reformistas (1870-1948), una época de activa producción científica en el país centroamericano y los autores elegidos fueron Henri Pittier, Karl Sapper, Dana Gardner Munro, Max Sorre, Rosa Filatti, Charles Borel y John y Mavis Biesanz, quienes brindaron aportes en la recopilación y estudio de información climática producida en el territorio costarricense.

\section{Generalidades del clima de Costa Rica}

Costa Rica se ubica en la región neotropical, al norte de la línea ecuatorial, en el istmo centroamericano y en medio del Océano Pacífico y del Mar Caribe; su clima se ve modificado por la acción de los vientos dominantes en su pequeño territorio de aproximadamente $51100 \mathrm{~km}^{2}$, atravesado por un sistema montañoso de noroeste a sureste con alturas que superan los 3500 metros (Mena, s.f., p.1). A grandes rasgos, esas son las condiciones que moldean el clima costarricense y para su mayor comprensión, el país ha sido dividido en seis regiones según sus similitudes en cuanto a distribución de precipitaciones y temperaturas: Valle Central, Pacífico Norte, Pacífico Central, Pacífico Sur, Vertiente del Caribe y Zona Norte.

\section{(C) $(000$}

La Revista Estudios es editada por la Universidad de Costa Rica y se distribuye bajo una Licencia Creative Commons Atribución-NoComercial-CompartirIgual 3.0 Costa Rica. Para más información envíe un mensaje a 
Dossier: La escritura en los desplazamientos: viajes, expediciones y naufragios Al ser el Valle Central la sección del país que alberga la mayor parte de la población, la infraestructura y por ser la sede de los poderes de la República y concentrar las principales actividades económicas, sociales y culturales desde la época colonial (Pérez, 1997, pp.13-31), es la región que más van a destacar los textos de los autores consultados y por tanto, la descripción de sus características climatológicas se generalizarán para todo el país, pese a las particularidades propia de cada una de sus zonas periféricas.

El clima del Valle Central - o bien, Meseta Central como lo llamaban los autores consultados - que comprende el centro del país, se caracteriza porque en la mayor parte del territorio que lo integra, posee una estación seca que coincide con el invierno boreal (diciembre a marzo) y una estación lluviosa que comprende del mes de mayo al de octubre, siendo los meses de abril y mayo períodos de transición de una estación a otra; experimenta una disminución de las precipitaciones en julio (veranillo de San Juan) y varía de una a tres semanas por un cambio en la circulación del viento. La temperatura oscila entre los $18^{\circ} \mathrm{C}$ y los $30^{\circ} \mathrm{C}$ como mínimos y máximos promedios, aunque el promedio se ubica entre los $22^{\circ} \mathrm{C}$ y los $24^{\circ} \mathrm{C}$ (Mena, s.f., pp.1-2).

En cuanto a los vientos, en la época seca predominan los alisios del Noreste con velocidades de hasta 30 kilómetros por hora entre febrero y marzo y en la lluviosa, dichos vientos disminuyen su intensidad. En horas de la mañana, inicia la penetración de la brisa del Pacífico con dirección Oeste - Noroeste que forma un frente de brisa que provoca la aparición de nubes de tipo cúmulo que logran un importante desarrollo vertical, causantes de aguaceros y tormentas en la región (Mena, s.f., p.3).

\section{(๑) $\odot \odot$}

La Revista Estudios es editada por la Universidad de Costa Rica y se distribuye bajo una Licencia Creative Commons Atribución-NoComercial-CompartirIgual 3.0 Costa Rica. Para más información envíe un mensaje a 
Dossier: La escritura en los desplazamientos: viajes, expediciones y naufragios Bajo estas condiciones, los autores consultados describieron el clima del Valle Central, ya sea para fines científicos o informativos, pero independientemente de la formación académica de quien produce cada texto, dichos rasgos climáticos aparecerán en las obras que se analizan a continuación, en forma cronológica, a partir de cada una de las personas autoras seleccionadas.

\section{Henri Pittier}

El Dr. Henri Pittier Dormond (1857-1950), prestigioso científico suizo, fue uno de los fundadores y Director del Observatorio Meteorológico (1887-1888), Instituto Meteorológico Nacional (1888-1889) e Instituto Físico-Geográfico Nacional (18891904), gran impulsor de la Meteorología y de las investigaciones científicas en el país (Conejo, 1975; Eakin, 1999, pp.123-150; Solano, 1999, 176-188; Monge y Méndez, 2002), recopiló toda la información meteorológica elaborada por los científicos europeos que le precedieron y por los Directores de la Oficina de Estadística comprendiendo aproximadamente cuatro décadas de registros de observaciones atmosféricas.

A pesar del impulso que le dio a los estudios meteorológicos desde las instituciones que dirigió, Pittier (1888) no siempre valoró en forma positiva los esfuerzos de sus antecesores, por ejemplo, esta crítica generalizada hecha a sus investigaciones:

se distinguen especialmente por la ausencia de todo espíritu de continuidad, lo cual debe de atribuirse á que estas observaciones son obra de personas aisladas, de mucha dedicación y sin duda muy capaces, pero que han carecido de las aptitudes que exigen las investigaciones de este género (p.8).

Tales afirmaciones fueron refutadas por Solano (1999, pp.185-187) al demostrar que dichas observaciones se ajustaban a los parámetros internacionales de la

\section{(C) $(\otimes \odot$}

La Revista Estudios es editada por la Universidad de Costa Rica y se distribuye bajo una Licencia Creative Commons Atribución-NoComercial-CompartirIgual 3.0 Costa Rica. Para más información envíe un mensaje a 
Dossier: La escritura en los desplazamientos: viajes, expediciones y naufragios época, la participación de instituciones y de personas con formación científica en la producción de dichos datos y a la elaboración de información meteorológica continua en las décadas previas al ingreso de Pittier a Costa Rica.

Precisamente, la primera entrega de la serie de "Apuntaciones sobre el clima é hipsometría de la República de Costa Rica" fue la obra inicial de Pittier en el país y en ella expuso a grandes rasgos los principales elementos de la climatología del territorio costarricense, en el que definió las características climatológicas mensuales del Valle Central (1888, pp.12-13). Estas ya habían sido investigadas anteriormente por von Frantzius (1967, pp.19-20) en su "Climatología de Centro América", donde propone un modelo del comportamiento climático adaptado por Pittier para explicar la dinámica climatológica de Costa Rica.

De acuerdo con von Frantzius, el viento constituye el elemento fundamental del clima de la zona y las observaciones meteorológicas producidas en Heredia atestiguaban en forma categórica "la mudanza de la zona del alisio con el curso del sol. Este se halla en el cenit de San José y Heredia á mediados de abril y agosto" (1967, p.12) por lo que asocia este cambio de la circulación del viento con el curso aparente del Sol en la bóveda celeste.

Por desavenencias con las figuras políticas de la época, Pittier rescindió su contrato en 1904 y se trasladó a los Estados Unidos para laborar en el Departamento de Agricultura (Yacher, 2000, pp.4-10). Este hecho permitió el arribo de nuevas figuras en el plano de la Meteorología y de la ciencia en general, algunos de cuyos trabajos se expondrán a continuación, por lo que no se deben de tomar al pie de la letra las siguientes declaraciones de Tristán: "Pittier se retiró. Todo tomó un tinte de ruina y abandono" (1966, p.124) como si la ausencia de este funcionario hubiera significado el fin del desarrollo científico nacional.

\section{(ब) $(\Theta \odot$}

La Revista Estudios es editada por la Universidad de Costa Rica y se distribuye bajo una Licencia Creative Commons Atribución-NoComercial-CompartirIgual 3.0 Costa Rica. Para más información envíe un mensaje a 
Dossier: La escritura en los desplazamientos: viajes, expediciones y naufragios

\section{Karl Sapper}

Karl Sapper (1866-1945), geólogo alemán quien realizó investigaciones en el campo de la geología en América Central, fue Director temporal del Instituto FísicoGeográfico (1924) y había hecho un viaje por el istmo al finalizar en siglo XIX que incluyó una corta estadía en Costa Rica (1899) (Instituto Geográfico Nacional, 1989, p.21; Trejos, 1998, pp.49-50; Alvarado y Denyer, 2012). Al ingresar al país por tierra, procedente de Nicaragua, Sapper fue observando los diferentes paisajes hasta el Valle Central y conoció los rasgos de los distintos climas que caracterizaron los puntos del país que visitó.

Sapper (1998) describe de esta forma el clima de Alajuela:

El clima aquí, como en toda la meseta del medio de Costa Rica, - Ilana, inclinada y surcada de vegas de ríos-, relativamente seco; es decir, se distingue por un largo período de sequía porque lo protegen del viento los enormes volcanes (p.68).

Además de permanecer en el centro del país, Sapper (1998) ascendió los volcanes de la Cordillera Volcánica Central y se adentró en los territorios indígenas de Chirripó, Talamanca y la Zona Norte del país, con lo que amplía el horizonte de estudios climáticos a pesar de que el objetivo primordial era la geología y etnografía de las regiones visitadas.

Un ejemplo de una descripción de los efectos del clima en una zona periférica de Costa Rica es el del aumento de los caudales de los ríos a causa de las lluvias en los territorios donde la estación seca prácticamente no existe (Mena, s.f., pp.8-10) y específicamente, del río Chirripó en la vertiente del Caribe, en el que Sapper

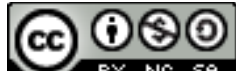

La Revista Estudios es editada por la Universidad de Costa Rica y se distribuye bajo una Licencia Creative Commons Atribución-NoComercial-CompartirIgual 3.0 Costa Rica. Para más información envíe un mensaje a 
Dossier: La escritura en los desplazamientos: viajes, expediciones y naufragios (1998) retomó una experiencia vivida por el obispo Bernardo Augusto Thiel (18501901), una década antes de su visita:

Aunque la corriente no era fuerte, a causa de la disminución del agua en esta época [marzo de 1899], pasamos sin dificultad la orilla opuesta, pero nos alegramos mucho de haber dejado atrás este peligroso río, pues un solo aguacero de mediana duración e intensidad aumenta su caudal de agua de tal manera que se hace muy peligroso, cuando no imposible, el atravesarlo (p.92).

De esta forma, los primeros autores analizados fueron científicos con formación en las ciencias naturales, quienes exploraron el territorio costarricense para identificar sus recursos y se ligaron a la institucionalidad científica en el marco de lo que Viales y Clare denominan "régimen de cientificidad" (2009, pp.97-109), que hizo posible el desarrollo de la ciencia en Costa Rica durante gran parte del período en estudio.

\section{Dana Gardner Munro}

El diplomático e historiador estadounidense Dana Gardner Munro (1892-1990), hijo del historiador Dana Carleton Munro (1866-1932) (Quesada, 2001, pp.625-626), visitó Costa Rica entre finales de 1914 e inicios de 1915 como parte de su investigación para una tesis referente a las causas de los conflictos políticos centroamericanos y publicada bajo el título The Five Republics in Central America (Munro, 1918).

La obra de Munro (1918) fue consultada por Sapper (Dobles, 1928, p.483) por la publicación de datos recientes referentes a las condiciones políticas, económicas y sociales por las que atravesaba el istmo centroamericano durante la primera posguerra mundial. La versión en español se titula Las cinco repúblicas de Centro América y fue publicada casi un siglo después (Munro, 2003).

\section{(c) (i) (-)}

La Revista Estudios es editada por la Universidad de Costa Rica y se distribuye bajo una Licencia Creative Commons Atribución-NoComercial-CompartirIgual 3.0 Costa Rica. Para más información envíe un mensaje a 
Dossier: La escritura en los desplazamientos: viajes, expediciones y naufragios Munro (1918, p.1) destaca el hecho que el territorio centroamericano, a pesar de su superficie relativamente pequeña, comprende muchas regiones con diversidad de climas, debido a la presencia de un relieve montañoso que atraviesa la mayor parte de su territorio, factor que repercute en las variaciones climáticas, que encierra mesetas y elevados valles donde los calores tropicales son atenuados por los efectos de la altitud.

Sin embargo, los datos que proporciona Munro (1918) sobre la climatología centroamericana son bastante escuetos, por el énfasis que hace en los aspectos políticos en su trabajo. Este autor describe al istmo como "una estrecha franja de tierra entre los Océanos Atlántico y Pacífico, extendiéndose del este y sur de México al istmo de Panamá" (p.1), cuya área de 171900 millas cuadradas, constituyen una superficie menor que la del estado de California (Munro, 2003, p.51).

La amplia llanura litoral que bordea el Caribe centroamericano era escasamente poblada hasta años recientes, debido a la intensa humedad y a las elevadas temperaturas, pero que desde finales del siglo XIX constituía un foco de relativo desarrollo económico por ser el asiento de las operaciones monopolísticas del enclave bananero (Munro, 1918, p.1).

Munro atribuye la fertilidad del suelo centroamericano a la combinación de varios elementos, como lo son la actividad volcánica, la benevolencia del clima de los valles intermontanos y la existencia de una estación lluviosa de copiosas pero no excesivas precipitaciones. Estas condiciones propiciaban las actividades agrícolas que daban alimento y trabajo a la mayor parte de la población centroamericana (Munro, 1918, p.2).

\section{(๑) (๑)}

La Revista Estudios es editada por la Universidad de Costa Rica y se distribuye bajo una Licencia Creative Commons Atribución-NoComercial-CompartirIgual 3.0 Costa Rica. Para más información envíe un mensaje a 
Dossier: La escritura en los desplazamientos: viajes, expediciones y naufragios Los valles de terrenos fértiles ubicados en el interior del istmo son el asiento de las principales ciudades centroamericanas, a una altitud entre los dos mil y siete mil pies, donde la temperatura rara vez excedía los ochenta grados en la escala Fahrenheit. Otro importante sector que concentraba buena parte de la población centroamericana eran las llanuras cálidas y secas que bordean la costa pacífica y culminan al pie de la cordillera volcánica (Munro, 1918, p.2) y que hoy día se les incluye en el denominado Corredor Seco Centroamericano (Quesada, Calvo, Hidalgo, Pérez y Alfaro, 2019).

En relación con Costa Rica, Munro (1918, pp.138-139 y pp.162-163) describió una población concentrada básicamente en "la fresca y fértil Meseta Central", centro del acontecer político y social de la nación, rodeada de volcanes y sierras de la Cordillera Centroamericana; salpicada de pequeñas propiedades dedicadas al sostenimiento de las familias campesinas y a la producción de café, que vendían a los propietarios de los beneficios para su procesamiento y exportación. Los habitantes del Valle Central, según Munro, "nunca han mostrado inclinación para expandirse dentro del montañoso país [Costa Rica] hacia el sur, donde la comunicación con los poblados sería bastante difícil, o en las cálidas e insalubres regiones costeras", por lo que permanece concentrada en este espacio dedicada principalmente a la economía cafetalera (Munro, 1918, pp.138-139).

No obstante, en esos mismos años, el Estado costarricense fomentaba la colonización de las zonas periféricas por medio de un proceso de autoinmigración, con la finalidad que el propio campesinado nacional ocupara dichas tierras y las integraran a la dinámica productiva agroexportadora (Palmer, 1995).

De acuerdo con Munro (1918), el carácter montañoso del territorio y los seis meses de duración de la estación lluviosa no eran obstáculo suficiente para que Costa Rica 
Dossier: La escritura en los desplazamientos: viajes, expediciones y naufragios careciera en ese momento de una razonable red de caminos, pero que debía ser mejorada (pp.138-139, p.157 y p.160).

En las postrimerías de su vida, Munro (1983) publicó un texto titulado A Student in Central America, 1914-1916, luego traducido al español (Munro, 2001), en el que relata sus experiencias como estudiante en los países del istmo centroamericano y donde anotó varias referencias alusivas al clima de los territorios visitados. En el caso de Costa Rica, el mencionado autor deja constancia en el siguiente pasaje sobre el efecto de los fenómenos atmosféricos en la vida cotidiana de los costarricenses de la época:

En setiembre y octubre se hacía difícil viajar fuera de la ciudad [San José]. Los aguaceros eran más fuertes, a veces comenzaban por la mañana y duraban todo el día, lo cual era a menudo desagradable. A veces no había electricidad; había apagones en las casas y las fiestas en el club de golf tenían que suspenderse porque el tranvía no funcionaba. Los aguaceros paraban el servicio del ferrocarril de modo que no llegaba el correo del extranjero, y varios empleados de la [United] Fruit Company que habían llegado a San José en vacaciones cortas estaban sin poderse ayudar en la ciudad. Lo peor de todo es que no había retretas. Como a mediados de noviembre de pronto pararon las lluvias y comenzó a ponerse fresco por las mañanas y por las noches, mientras que el mediodía era caluroso. (Munro, 2001, p.646)

Esta obra expone una visión sintética del clima centroamericano, engloba a grandes rasgos sus características generales, destaca la diversidad climática como un producto de las diferencias de altitud por el relieve y la presencia de una estación lluviosa de significativa duración. Sin embargo, Munro (1983, 2001) no profundizó en las causas que producen dicha variedad de climas ni ofreció datos sobre observaciones meteorológicas en lugares específicos, ni tampoco se cuestiona sobre la continuidad histórica de las relaciones entre el clima y los principales asentamientos humanos en el istmo, al parecer, por carecer de la formación debida para dicho análisis y por centrar su visión en la situación política del área.

\section{(c) (i) (2)}

La Revista Estudios es editada por la Universidad de Costa Rica y se distribuye bajo una Licencia Creative Commons Atribución-NoComercial-Compartirlgual 3.0 Costa Rica. Para más información envíe un mensaje a 
Dossier: La escritura en los desplazamientos: viajes, expediciones y naufragios

Este énfasis en lo político puede atribuirse a la creciente expansión económica y política que caracterizó a los Estados Unidos al finalizar el siglo XIX, que lo llevó a practicar intervenciones militares en las débiles repúblicas del área durante los primeros años del siglo XX (Munro, 1918, p.76, pp.95-96, y pp.303-319; Sibaja, 1974, pp.210-211).

\section{Max Sorre y Rosa Filatti}

El geógrafo francés Max Sorre (1880-1962) y su colega mexicana Rosa Filatti (18911943), redactores del volumen sobre México y América Central de la Geografía universal (1930), dirigida por los geógrafos franceses Paul Vidal de la Blache (18451918) y Lucien Gallois (1857-1941), destacaron la gran variedad de climas presentes en Costa Rica, gracias a su orografía y lo demuestran haciendo un análisis de la climatología costarricense según las regiones en que dividieron el país: Llanuras del Norte y del Este, zonas montañosas, Meseta Central y Vertiente del Pacífico (Sorre y Filatti, 1948, pp.259-271).

Uno de los análisis más detallados de Sorre y Filatti (1948) es el que corresponde al clima de la Meseta Central, donde residía la mayor parte de la población costarricense, en el que se aborda la influencia de los vientos en la conformación del clima de la zona y las modificaciones que a su vez se producen a una escala subregional al evidenciar distintos tipos de condiciones climáticas en la parte central del país y que se transcribe a continuación:

El clima y el trazado de la red hidrográfica acusan la concurrencia de las influencias atlánticas y pacíficas. Los vientos entre el Este y el Nordeste, partiendo del Mar Caribe, remontan las pendientes de la Sierra de Talamanca y el valle del Reventazón. Mas la mayor parte de la meseta se encuentra substraída a su influencia, abrigada como está por la cordillera

\section{(๑) $\odot \odot$}

La Revista Estudios es editada por la Universidad de Costa Rica y se distribuye bajo una Licencia Creative Commons Atribución-NoComercial-CompartirIgual 3.0 Costa Rica. Para más información envíe un mensaje a revistaestudios.eeg@ucr.ac.cr. 


\section{Dossier: La escritura en los desplazamientos: viajes, expediciones y naufragios} volcánica; sufre el régimen de vientos estacionales del Pacífico (vientos de Sudoeste). También la línea de repartición climática está al Este de la línea de partición hidrográfica. Cuando se remonta el Reventazón hasta Juan Viñas (1.181 metros) se está en el dominio de las lluvias persistentes con máxima de invierno; pero en Cartago (1.451 metros) y Aguacaliente, en la misma cuenca, reinan lluvias de principios del verano y de otoño con inviernos secos, como en todas las vertientes del Pacífico. Tal es el régimen de la meseta de San José. A 1.160 metros de altura, el período seco dura como cinco meses, de diciembre a marzo; es también el período menos caliente. En abril comienzan las lluvias, que duran hasta noviembre, con una mínima secundaria en julio, apenas marcada. Su llegada modera y en seguida detiene el ascenso del termómetro, de suerte que, entre la media del mes más caliente (mayo) y la del mes más frío (enero), la diferencia es solamente de 107 para un promedio de 19\%6. Las precipitaciones proporcionan 1.984 milímetros por año. Se producen diariamente a la misma hora, entre la una y las cuatro de la tarde, después de una mañana serena (pp.265-266).

La anterior descripción del clima del Valle Central de Costa Rica detalla la influencia de los vientos procedentes de ambos océanos, siendo predominante la de los alisios del Atlántico en la sección más oriental de la cuenca del río Reventazón, mientras que el resto de la región se halla expuesta a la influencia de los oestes sinópticos procedentes del Pacífico. Es curioso apuntar que ambos autores utilizan los conceptos de las estaciones astronómicas para referirse al clima de este espacio y la ausencia de oscilaciones fuertes en los promedios de temperatura entre el mes más cálido y el más frío.

Los autores observaron también, a partir del mapa de Costa Rica elaborado bajo la dirección de Pittier, la desventaja del río Grande con respecto al Reventazón por la menor cantidad de lluvias que aumentan su caudal (Sorre y Filatti, 1948, p.266) ya que el segundo, recibía más cantidad de agua al estar más expuesto a los alisios provistos de humedad que penetran por el Valle del Reventazón, a diferencia del primero, uno de los principales ríos de la vertiente pacífica.

\section{(c) (i) (-)}

La Revista Estudios es editada por la Universidad de Costa Rica y se distribuye bajo una Licencia Creative Commons Atribución-NoComercial-CompartirIgual 3.0 Costa Rica. Para más información envíe un mensaje a 
Dossier: La escritura en los desplazamientos: viajes, expediciones y naufragios

\section{Charles Borel}

El profesor y físico suizo Charles E. Borel Lauterburg (1896-1992), quien ingresó a Costa Rica en 1929, en compañía de los científicos Paul Schaufelberger (18941976), Jan Wakulski y Louis Appel, para laborar como profesores de las asignaturas científicas en el Liceo de Costa Rica (Porras, 1988, p.432). Por medio de esta contratación, se podría inferir que el Gobierno quería revitalizar la comunidad científica nacional con el fin de incorporar las teorías y experiencias con los que estos científicos fueron formados y promover nuevas investigaciones sobre los recursos naturales del territorio costarricense.

Este Profesor de Física, conocedor de los nuevos paradigmas en la investigación de la dinámica de la atmósfera, incentivó al joven José Merino y Coronado (19121987), a estudiar el comportamiento de los fenómenos atmosféricos con base en la recepción de ondas de radio procedentes del exterior y cuyo fin era monitorearlas para efectuar pronósticos del estado del tiempo (Borel y Merino, 1934, pp.235-241). Sin duda, un esfuerzo por modernizar la ciencia meteorológica y ofrecer nuevos enfoques que superaran los estudios meramente descriptivos del clima de los distintos espacios que componen el territorio nacional.

Borel (1934a, p.234), escribió un artículo titulado "Datos meteorológicos del país", en el que se resumía el estado de la meteorología costarricense a mediados de la década de 1930, donde persisten algunas continuidades de los estudios de las décadas previas: centralismo de las observaciones en San José, observatorio localizado en la vieja torre del antiguo edificio del Liceo de Costa Rica y control de la compañía bananera de la red de estaciones pluviométricas que pertenecía al Instituto Físico-Geográfico Nacional.

\section{(C) $(000$}

La Revista Estudios es editada por la Universidad de Costa Rica y se distribuye bajo una Licencia Creative Commons Atribución-NoComercial-CompartirIgual 3.0 Costa Rica. Para más información envíe un mensaje a 
Dossier: La escritura en los desplazamientos: viajes, expediciones y naufragios Este autor reseñó la situación de la Meteorología en la Costa Rica de su tiempo en el siguiente fragmento, donde se muestra la difusión de la información meteorología producida en el país en prestigiosas revistas extranjeras Borel (1934a):

Hay [en Costa Rica] un servicio meteorológico oficial (Torre al sur del Museo Nacional). Ahí se registran los elementos del tiempo para San José. Estos se publican en el "Diario de Costa Rica" y en el anuario estadístico.

Unos finqueros hacen medidas de la lluvia y de la temperatura. El servicio más completo de la república es el de la United Fruit Co. Registra la cantidad de lluvias, las temperaturas extremas y la humedad en un gran número de fincas de la zona atlántica y del valle del Reventazón. Unos de estos datos aparecen a intervalos irregulares en "Climatological Date, West Indies and Caribbean Service", publicado por el Departamento de Agricultura de los Estados Unidos (p.234).

Una novedad de esta publicación es la inclusión de promedios mensuales de precipitación para diecinueve estaciones, algunas de ellas pertenecientes a la red pluviométrica vinculada al enclave bananero (Guápiles, Limón, Boca Banano, Peralta, Turrialba y Juan Viñas), recopilados a partir de los datos publicados por el Instituto Físico-Geográfico en sus primeras etapas, por el Boletín de Fomento y otros inéditos (Borel, 1934a, p.234). Las demás estaciones - muchas de ellas iniciaron la recolección de datos a finales del siglo XIX - eran las de San José, Sarapiquí, Toro Amarillo, Cariblanco, Sipurio, Cartago, Tres Ríos, La Uruca, Nuestro Amo, Puntarenas, Las Cañas, Filadelfia y la de San Juan del Norte (Nicaragua).

Borel (1934b) elaboró otro artículo, "El clima de San José" en los que demuestra su conocimiento de la climatología del Valle Central, en particular del espacio que ocupa la ciudad capital y los factores que hacen de él un clima bondadoso con una temperatura media anual similar a las de "San Diego de California, de Savannah [Georgia, Estados Unidos] sobre la costa atlántica o de Túnez, en la costa sur del Mediterráneo" (p.242).

\section{() $(\Theta \odot$}

La Revista Estudios es editada por la Universidad de Costa Rica y se distribuye bajo una Licencia Creative Commons Atribución-NoComercial-CompartirIgual 3.0 Costa Rica. Para más información envíe un mensaje a 
Dossier: La escritura en los desplazamientos: viajes, expediciones y naufragios La temperatura del mes más frío (18,8ㅜㅡ en diciembre) al más cálido (20,5ํe en mayo) en San José oscilaba entre $1,7^{\circ} \mathrm{C}$ anuales y a pesar de presentar variaciones diurnas de $10^{\circ}$ en diciembre y $15^{\circ}$ en mayo, estas no eran bruscas gracias al efecto föhn que experimentaba la nubosidad que penetraba en el Valle Central por los pasos de La Palma y El Desengaño en la Cordillera Volcánica Central (al norte de la ciudad). Se debe considerar también como factores que intervienen en este proceso la condensación del exceso de humedad que permite al aire conservar en parte su alta temperatura, la acción de la brisa del valle en el día y de montaña en la noche, la nebulosidad relativamente abundante en el cielo de la capital y la acción directa de la Zona de Convergencia Intertropical (ZCI) en su paso por Costa Rica durante el verano boreal (Borel, 1934b, p.242).

La introducción de conceptos como los de brisa de valle y montaña y el de la Zona de Convergencia Intertropical, constituyen una novedad en los estudios climatológicos nacionales, al destacar la incidencia de factores locales y regionales que modifican el comportamiento del clima. A pesar de ello, el Valle Central y específicamente la ciudad capital, concentraban la mayor parte de los análisis referentes al clima costarricense.

\section{John y Mavis Biesanz}

Al final del período seleccionado para la presente investigación, merece especial atención, respecto al clima de Costa Rica, la obra Costa Rican Life (1944) de los sociólogos estadounidenses John Biesanz (1913-1995) y Mavis Hiltunen Biesanz (1919-2008), cuya versión en español se publicaría tres decenios más tarde con el título La vida en Costa Rica (Biesanz y Biesanz, 1975). Ambos autores residieron en la ciudad de Heredia durante la primera mitad de la década de 1940, mientras se dedicaban a recopilar información sobre el territorio, la sociedad y la cultura de

\section{(C) $(0 \bigcirc$}

La Revista Estudios es editada por la Universidad de Costa Rica y se distribuye bajo una Licencia Creative Commons Atribución-NoComercial-CompartirIgual 3.0 Costa Rica. Para más información envíe un mensaje a 
Dossier: La escritura en los desplazamientos: viajes, expediciones y naufragios los ticos en los años previos al conflicto armado de 1948, para difundir el resultado de sus observaciones en los Estados Unidos con fines turísticos (Biesanz y Biesanz, 1944, p.2).

Al ser este el enfoque, los Biesanz (1975) muestran una imagen idealizada del país, al cual llegan a identificarlo con el fértil Valle Central, la "Tierra de la Eterna Primavera", donde los cafetales constituían el elemento principal del paisaje. Esta idealización se extiende al sistema político y social costarricense (Biesanz y Biesanz, 1944, pp.2-3 y pp.17-18).

En opinión de ambos, el Valle Central costarricense posee "uno de los climas imaginables más agradables [del mundo]", ya que San José reportaba una temperatura promedio de $70^{\circ} \mathrm{F}$, afectada por una variación anual de $5^{\circ}$ en dicha escala, gracias a la altura en que se encontraba, porque destacan que la capital se sitúa a la reducida distancia de 625 millas del ecuador, en una latitud similar al extremo sur de la India y a la porción norte de Venezuela (Biesanz y Biesanz, 1944, pp.2-3).

Las zonas costeras, eran descritas como calientes y húmedas, propicias para el cultivo del banano, el cacao y aquellos que fueron impulsados para abastecer el consumo del mercado de los Estados Unidos en tiempos de la Segunda Guerra Mundial (1939-1945): caucho, quina y cáñamo (Biesanz y Biesanz, 1944, p.3).

Ambos autores indicaron que paralela a la floración del cafeto, entre abril y mayo, comienza la estación lluviosa que transforma al Valle Central en un "exuberante jardín verde", hasta su conclusión en el mes de noviembre. En el siguiente fragmento se expone su percepción del estado del tiempo durante el período de

\section{(C) $(000$}

La Revista Estudios es editada por la Universidad de Costa Rica y se distribuye bajo una Licencia Creative Commons Atribución-NoComercial-CompartirIgual 3.0 Costa Rica. Para más información envíe un mensaje a 
Dossier: La escritura en los desplazamientos: viajes, expediciones y naufragios lluvias en dicha región del país y su incidencia en la cotidianidad de sus moradores (Biesanz y Biesanz, 1944):

Excepto por ocasionales períodos de lluvia continua, el cielo es azul y el sol brilla hasta el medio día o más tarde, cuando comienzan el diario aguacero vespertino. En algunos meses, estas lluvias son tan fuertes y continúan hasta el anochecer. Una sombrilla es un accesorio permanente de todos los costarricenses durante esta estación. Desde el conductor descalzo de carretas con bueyes a la elegante señora de la capital. La estación seca es llamada "verano" - y en un día soleado durante la estación lluviosa, el comentario general sobre el tiempo dice que "hace verano" (pp.3-4 [compárese Biesanz y Biesanz, 1975, p.25]).

Los autores elogian la benignidad del clima del Valle Central, ideal para los extranjeros por sus días agradables y noches frescas, por lo que la ausencia de inviernos nevados, favorece la "facilidad" con que el costarricense vive su vida. A pesar de su variedad climática, el país carecía de huracanes y calores extremos y siguiendo la creencia popular, asocian la ocasional sismicidad de marzo y diciembre con los cambios de estación (Biesanz y Biesanz, 1944, p.4).

Los leves cambios en la temperatura, sin embargo, tienen sus consecuencias para los costarricenses, de acuerdo con Biesanz y Biesanz:

Cuando el aire es solo unos pocos grados más frío de lo usual, en las madrugadas o después de un aguacero - ellos [los costarricenses] tiemblan y se quejan, 'Ay que frio! [sic]' (How cold it is!). Y si hay una sospecha de más calor que el usual, se abanican suavemente y exclaman a intervalos frecuentes, 'Qué calor! [sic]' (How warm!)." (1944, p.4 [compárese Biesanz y Biesanz, 1975, p.25]).

De esta forma se analizaron aspectos del clima en el espacio geográfico ocupado por la República de Costa Rica, descritos por ocho autores que visitaron o investigaron sobre este aspecto del país en un período que abarcó casi seis

\section{(๑) $\odot \odot$}

La Revista Estudios es editada por la Universidad de Costa Rica y se distribuye bajo una Licencia Creative Commons Atribución-NoComercial-CompartirIgual 3.0 Costa Rica. Para más información envíe un mensaje a 
Dossier: La escritura en los desplazamientos: viajes, expediciones y naufragios décadas, donde es posible identificar los avances adquiridos en el conocimiento de la atmósfera que fueron estudiados por las instituciones científicas de la época.

\section{Conclusiones}

Los escritos de los naturalistas, científicos sociales y otros especialistas a lo largo del período de investigación comprendido en el presente artículo permitieron el análisis de la percepción que tenían del clima costarricense, al ser publicadas o distribuidas fuera de Costa Rica. De esta forma, la comunidad científica internacional, los comerciantes y los visitantes procedentes del exterior podían darse una idea de las condiciones climáticas en el país centroamericano.

Es significativo que mucho antes de la fundación del Observatorio Meteorológico del Liceo de Costa Rica (1887) el comportamiento del clima de Costa Rica ya había sido abordado por von Frantzius y sus observaciones fueron de utilidad para los trabajos de Pittier, cuya entidad siguió recopilando la información meteorológica producida en el país y fue complementada con los datos procedentes de científicos de distintas formaciones que llegaron a atribuir al sistema de vientos y al relieve la variedad climática existente en el territorio costarricense.

Los trabajos producidos entre 1888 y 1944 coinciden desde el punto de vista cronológico con una época que para la historiografía costarricense de la Historia de la Ciencias tradicional, denomina "aislacionista" y que comprendería de 1904 a 1940 (desde la renuncia de Pittier a la fundación de la Universidad de Costa Rica) caracterizada por "la acción fundamental de científicos costarricenses, con formación científica (o sin ella), y en algunos casos con el apoyo de investigadores extranjeros que visitan el país" (Coronado, 1997, p.263).

\section{(c) (i) (-)}

La Revista Estudios es editada por la Universidad de Costa Rica y se distribuye bajo una Licencia Creative Commons Atribución-NoComercial-CompartirIgual 3.0 Costa Rica. Para más información envíe un mensaje a revistaestudios.eeg@ucr.ac.cr. 
Dossier: La escritura en los desplazamientos: viajes, expediciones y naufragios No obstante, la producción científica relacionada con el conocimiento meteorológico durante este período fue obra de una comunidad científica, fue difundida en el extranjero y analizada científicamente en obras como la de Sorre y Filatti (1948), lo que permitió tener un conocimiento más pormenorizado del comportamiento de la atmósfera en Costa Rica, en el marco del "régimen de cientificidad" propuesto por Viales y Clare (2009), que implicaba la participación de diversos actores que hicieron posible la investigación científica cuyos productos fueron dados a conocer en el extranjero.

Las descripciones y análisis que los autores ofrecen no difieren a grandes rasgos y son similares a las actuales condiciones climatológicas, aspecto que deberá ser estudiado con mayor detalle por investigaciones futuras que permitan dilucidar las continuidades y discontinuidades atribuibles al cambio y a la variabilidad climática presentes en el territorio costarricense.

Cabe señalar la influencia de la formación profesional de los autores en los textos analizados: quienes proceden de las ciencias naturales (Pittier, Sapper, Sorre, Filatti y Borel), ofrecían cuadros explicativos para comprender la dinámica del clima de Costa Rica y aportaban datos cuantitativos relacionados con el comportamiento de la atmósfera, a diferencia de los procedentes de las ciencias sociales (Munro, Biesanz y Biesanz) que enfatizaron más los aspectos culturales, sociales y políticos de la realidad costarricense y por tanto, describieron la incidencia entre los fenómenos atmosféricos en la cultura y sociedad de este país centroamericano.

Al ser el Valle Central el centro neurálgico de las funciones políticas, económicas, sociales y culturales del país, va a concentrar la mayor parte de las menciones del clima que aparecen en los textos investigados. Sin embargo, autores como Sapper estudiaron las condiciones climáticas propias de las zonas periféricas, que estaban 
Dossier: La escritura en los desplazamientos: viajes, expediciones y naufragios en la mente de los políticos y empresarios de la época, quienes las representaban como una fuente inagotable de recursos cuya explotación favorecería el progreso económico de la nación costarricense. En futuras investigaciones, sería conveniente contar con estudios a nivel nacional sobre las percepciones y análisis del clima en los que se incorpore una perspectiva regional e histórica abordando la mayor cantidad de fuentes posibles para tal fin.

Los estudios analizados ubicados en el período 1888-1944 muestran un conocimiento del clima en el territorio costarricense, ya sea desde la experiencia personal o bien, a partir de la consulta de fuentes anteriores o en ambos casos, no carente de dinamismo y rigurosidad científica y por ello, sería necesario efectuar el análisis de los textos de otros autores y autoras producidos en este período, para contribuir con el desarrollo de la Historia del Clima, la Meteorología y las Ciencias en Costa Rica, mediante el acopio de la información recopilada y su comparación con datos procedentes de otras fuentes, especialmente las elaboradas por las instituciones científicas relacionadas con la ciencia meteorológica.

\section{Agradecimiento}

Este artículo se elaboró en el marco del Programa de Estudios Sociales de la Ciencia, la Técnica y el Medio Ambientes (PESCTMA, 805-A4-906) del Centro de Investigaciones Geofísicas (CIGEFI) de la Universidad de Costa Rica. Avances del mismo fueron presentados en el Segundo Encuentro de Investigadores Jóvenes en la Estación Biológica Palo Verde, Costa Rica, del 1 al 3 de noviembre de 2002 y en el Congreso Internacional "La escritura en el desplazamiento: viajes, expediciones y naufragios" organizado por la Universidad de Costa Rica del 21 al 25 de octubre de 2019. El autor agradece la colaboración de Gabriel Madriz-Sojo en la revisión del texto en su versión final.

\section{(C) $(\Theta \odot \odot$}

La Revista Estudios es editada por la Universidad de Costa Rica y se distribuye bajo una Licencia Creative Commons Atribución-NoComercial-CompartirIgual 3.0 Costa Rica. Para más información envíe un mensaje a revistaestudios.eeg@ucr.ac.cr. 
Dossier: La escritura en los desplazamientos: viajes, expediciones y naufragios

\section{Bibliografía}

Alvarado, G. y Denyer, P. (ed.). (2012). Karl T. Sapper (1866-1945). Geólogo pionero en América Central. San José: EUCR.

Biesanz, J. y Biesanz, M. (1944). Costa Rican Life. New York: Columbia University Press.

Biesanz, J. y Biesanz, M. (1975). La vida en Costa Rica. San José: Ministerio de Cultura, Juventud y Deportes.

Borel, C. (1934a). Datos meteorológicos del país. En Bergna, L. y Zen, A. (ed.), Anuario General de Costa Rica. 1934 (p.234). San José: Imprenta Borrasé Hnos.

Borel, C. (1934b). El clima de San José. En Bergna, L. y Zen, A. (ed.), Anuario General de Costa Rica. 1934. (pp. 242-244). San José: Imprenta Borrasé Hnos.

Borel, C., y Merino, J. (1934). La Predicción del Tiempo mediante el Radio. En Bergna, L. y Zen, A. (ed.), Anuario General de Costa Rica 1934 (pp.235-241). San José: Imprenta Borrasé Hnos.

Conejo, A. (1972). Materiales para una bio-bibliografía costarricense del Dr. Henri Pittier Dormond (Tesis de Licenciatura). Universidad de Costa Rica, San José, C.R.

Conejo, A. (1975). Henri Pittier. San José: Ministerio de Cultura, Juventud y Deportes.

Coronado, G. (1997). La actividad científica en Costa Rica: bosquejo de su evolución. En Zamora, A. (Compilador), El otro laberinto (tecnología, filosofía, historia) (pp. 257-276). Cartago: Editorial Tecnológica de Costa Rica.

Díaz, Ronald. (2003). El proceso de institucionalización de la meteorología en Costa Rica (1887-1949) (Tesis de Licenciatura). Universidad de Costa Rica, San José, C.R.

Dobles, L. (1928) Índice bibliográfico de Costa Rica, Tomo II. San José: Imprenta Lehmann.

Eakin, M. (1999). The origins of modern science in Costa Rica: The Instituto FísicoGeográfico Nacional, 1887-1904. Latin American Research Review 34(1), 123-150.

\section{(c) $($ ) (2) (2)}

La Revista Estudios es editada por la Universidad de Costa Rica y se distribuye bajo una Licencia Creative Commons Atribución-NoComercial-CompartirIgual 3.0 Costa Rica. Para más información envíe un mensaje a revistaestudios.eeg@ucr.ac.cr. 
Dossier: La escritura en los desplazamientos: viajes, expediciones y naufragios

Frantzius, A. von (1967). Climatología de Centroamérica. Revista Conservadora del Pensamiento Centroamericano 79, 1-23.

Hilje, L. (2013). Trópico agreste. La huella de los naturalistas alemanes en la Costa Rica del siglo XIX. Cartago: Editorial Tecnológica de Costa Rica.

Instituto Geográfico Nacional. (1989) Cien años al servicio del país: 1889-1989. San José: IGN.

Mena, M. (s.f.). Mimeo sobre el clima de Costa Rica. San José: Gestión de Información, Instituto Meteorológico Nacional, Ministerio de Energía y Minas.

Monge, J. y Méndez, V. (2002). Dos colosos de la biología costarricense del siglo XIX: Anastasio Alfaro y Henri Pittier. En Peraldo, G. (Compilador), Ciencia y Técnica en la Costa Rica del siglo XIX (pp. 323-343). Cartago: Editorial Tecnológica de Costa Rica.

Munro, D. (1918). The Five Republics of Central America. New York: Oxford University Press.

Munro, D. (1983). A Student in Central America, 1914-1916. New Orleans: Middle American Research Institute, Tulane University.

Munro, D. (2001). Un estudiante en América Central. En Quesada, M., Entre silladas y rejoyas. Viajeros por Costa Rica de 1850 a 1950 (pp. 626-649). Cartago: Editorial Tecnológica de Costa Rica.

Munro, D. (2003). La Cinco Repúblicas de Centroamérica. Desarrollo Político y Económico y las Relaciones con Estados Unidos. San José: EUCR.

Palmer, S. (1995). Hacia la 'auto-inmigración'. El nacionalismo oficial en Costa Rica. 1870-1930. En Taracena, A. y Piel, J., Identidades nacionales y Estado moderno en Centroamérica (pp. 75-85). San José: EUCR.

Pérez, H. (1997). Breve historia contemporánea de Costa Rica. México: FCE.

Pittier, H. (1888). Apuntaciones sobre el clima é hipsometría de la República de Costa Rica. I. Resumen de las observaciones meteorológicas anteriores al año de 1888. En Boletín trimestral del Instituto Meteorológico Nacional, 1(1-2), 8-13.

\section{(®) $\odot \odot$}

La Revista Estudios es editada por la Universidad de Costa Rica y se distribuye bajo una Licencia Creative Commons Atribución-NoComercial-CompartirIgual 3.0 Costa Rica. Para más información envíe un mensaje a 
Dossier: La escritura en los desplazamientos: viajes, expediciones y naufragios Porras, D. (1988). Liceo de Costa Rica. 100 años. 1887-1987. Álbum de recopilación fotográfica del centenario del Liceo de Costa Rica. San José: Trejos.

Quesada, L., Calvo, O., Hidalgo, H., Pérez, P. y Alfaro, E. (2019). Dynamical delimitation of the Central American Dry Corridor (CADC) using drought indices and aridity values. Progress in Physical Geography: Earth and Environment 43(5), 627642, DOI: doi.org/10.1177/0309133319860224.

Quesada, M. (2001). Dana Gardner Munro. En Quesada, M. (Compilador.), Entre silladas y rejoyas. Viajeros por Costa Rica de 1850 a 1950 (pp. 623-626). Cartago: Editorial Tecnológica de Costa Rica.

Sapper, K. (1998). Viajes y estudios centroamericanos. En Zeledón, E. (Compilador), Viajes por la República de Costa Rica: Seebach-Sapper (pp. 51-110), Vol. III. San José: Ministerio de Cultura, Juventud y Deportes y Museo Nacional de Costa Rica.

Secretaría de Instrucción Pública de la República de Costa Rica. (1889). Anales del Instituto Físico-Geográfico Nacional, II.1. San José: Tipografía Nacional.

Sibaja, L. (1974). Nuestro límite con Nicaragua. Estudio histórico. San José: Comisión Nacional de Conmemoraciones Históricas.

Solano, F. (1999). El proceso de institucionalización de la meteorología en Costa Rica en el siglo XIX (Tesis de Licenciatura). Universidad de Costa Rica, San José, C.R.

Solano, F., Díaz, R. y Amador, J. (2013). La institucionalización de la meteorología en Costa Rica (1860-1910). San José: Editorial Nuevas Perspectivas.

Sorre, M. y Filatti, R. (1948). Costa Rica y Panamá. En Vidal, P. y Gallois, L. (ed.) Geografía universal. México y América Central (pp.259-282), Tomo XVIII, 2ª ed. Barcelona: Montaner y Simón.

Trejos, J. (1998). Karl Sapper. En Zeledón, E. (Compilador), Viajes por la República de Costa Rica: Seebach-Sapper (pp.49-50), Vol. III. San José: Ministerio de Cultura, Juventud y Deportes y Museo Nacional de Costa Rica.

Tristán, F. (1966). Baratijas de antaño. San José: Editorial Costa Rica.

Viales, R. y Clare, P. (2009). El Estado, lo transnacional y la construcción de comunidades científicas en la Costa Rica liberal (1870-1930). La construcción de un

\section{(๑) $\odot \odot$}

La Revista Estudios es editada por la Universidad de Costa Rica y se distribuye bajo una Licencia Creative Commons Atribución-NoComercial-CompartirIgual 3.0 Costa Rica. Para más información envíe un mensaje a 
Dossier: La escritura en los desplazamientos: viajes, expediciones y naufragios 'régimen de cientificidad. En Viales, R., Amador, J., y Solano, F. (ed.), Concepciones y representaciones de la naturaleza y la ciencia en América Latina (pp.97-109). San José: Universidad de Costa Rica, Vicerrectoría de Investigación.

Yacher, L. (2000). Henri F. Pittier's Professional Contributions and the Status of Geography in Costa Rica after his Permanent Departure. Brenesia 53, 3-16.

\section{(c) (i) (2)}

La Revista Estudios es editada por la Universidad de Costa Rica y se distribuye bajo una Licencia Creative Commons Atribución-NoComercial-CompartirIgual 3.0 Costa Rica. Para más información envíe un mensaje a revistaestudios.eeg@ucr.ac.cr. 